\title{
In search of sun-climate connection using solar irradiance measurements and climate records
}

\author{
Richard K. Kiang" and H. Lee Kyle ${ }^{\dagger}$ \\ NASA Goddard Space Flight Center \\ Greenbelt, MD 20771
}

\begin{abstract}
The Earth's temperature has risen approximately 0.5 degree- $C$ in the last 150 years. Because the atmospheric concentration of carbon dioxide has increased nearly $30 \%$ since the industrial revolution, a common conjecture, supported by various climate models, is that anthropogenic greenhouse gases have contributed to global warming. Another probable factor for the warming is the natural variation of solar irradiance. Although the variation is as small as $0.1 \%$, it is hypothesized that it contributes to part of the temperature rise. Warmer or cooler ocean temperature at one part of the Globe may manifest as abnormally wet or dry weather patterns some months or years later at another part of the globe. Furthermore, the lower atmosphere can be affected through its coupling with the stratosphere, after the stratospheric ozone absorbs the ultraviolet portion of the solar irradiance. In this paper, we use wavelet transforms based on Morlet wavelet to analyze the timefrequency properties in several datasets, including the Radiation Budget measurements, the long-term total solar irradiance time series, the long-term temperature at two locations for the North and the South Hemisphere. The main solar cycle, approximately 11 years, are identified in the long-term total solar irradiance time series. The wavelet transform of the temperature datasets show annual cycle but not the solar cycle. Some correlation is seen between the length of the solar cycle extracted from the wavelet transform and the North Hemisphere temperature time series. The absence of the 11 -year cycle in a time series does not necessarily imply that the geophysical parameter is not affected by the solar cycle; rather it simply reflects the complex nature of the Earth's response to climate forcings.
\end{abstract}

Keywords: wavelet transform, global warming, climate change, greenhouse gases

\section{INTRODUCTION}

The Earth appears to becoming warmer. Climate records have shown that the temperature of the Earth has risen approximately $0.5^{\circ} \mathrm{C}$ in the last 150 years. Growing evidences, from the retreating glaciers to the early sprouting of the northern forests, are alerting us that global warming may indeed be occurring. What remains uncertain is the cause for this temperature increase. There have been considerable debates on whether the warming is due to the greenhouse gases from anthropogenic activities, or a natural variation of the earth system, or a combination of the two. The occurrence of EI Niño and La Niña did not make the debate any easier. While themselves natural oscillations in the Earth system that are somewhat periodic, the observed unusual weather patterns could also be a combination of these oscillations and global warming.

Because the sun provides the main energy input into the Earth system, the first question that should be asked is how much the changes are due to the Sun. Historically, the Sun is thought to have undergone larger changes. Notably. the Little lce Age approximately 300 years ago in Europe and North America peaked at the Maunder Minimum in solar activity. when the solar irradiance is estimated to have been about $0.25 \%$ lower than the current level ${ }^{1.2}$.

Even though the Sun's total radiation output has been so steady that it is often referred as solar constant in many practical calculations, the Sun, after all. is a variable star -- a star whose total radiation varies with time. Aside from the very noticeable. 11-year sunspot cycle. the Sun has displayed other periodicity. like the 80 to 90 years Gleissberg cycle. Spectrally the greatest solar variations occur in the UV and the X-ray region in the shorter wavelengths and the radio waves in the longwave region.

* Correspondence: richard.kiang $\underline{a g f c}$ nasa.gov: 'kyle â eosdata.gsfc.nasa.gov, retired.

To be presented at the SPIE Conterence on Wavelet Application VII. 26-28 April 2000, Orlando. 
The Nimbus-7 Earth Radiance Budget experiment" started taking measurements on November 16. 1978. The measurements continued through January 24, 1993 (when the sun drifted out of the sensor's field of wiew). with the only major gap accurring from June 17 to September 2. 1992. Accurate total solar irradiance measurements require a mean experimental uncertainty no greater than a few parts in 10,000. Due to atmospheric variability, particularly in composition (water vapor, haze, etc.), this can only be achieved from satellites. The accuracy of the Nimbus-7 measurements has been examined in details by Hoyt et al." Analysis of possible calibration errors indicated that the long term trend in the solar measurements should be accurate to $\pm 0.03 \%$. Some evidence indicates that it might be as good as $\pm 0.01 \%$. The data showed that the total solar irradiance dropped by 0.1 to $0.2 \%$ when large dark sun spot groups faced the Earth and it rose by a somewhat lesser amount when bright faculae faced the Earth. Perhaps other factors are also involved ${ }^{10}$. The annual mean solar irradiance varied roughly in phase with the 11 -year sun spot cycle. It was about $0.1^{\%}$ higher at sunspot maximum than at sunspot minimum.

Other independent experiments have verified the solar variability. Measurements from the Solar Maximum Mission (SMM) satellite are available from February 16, 1980 to the summer of $1989^{10}$. The Earth Radiation Budget Satellite (ERBS) started solar measurements on October $25,1984^{\prime \prime}$. Its regular measurement program continued to the summer of 1993 when battery problems began to reduce the number of allowable measurements. The accuracy of the absolute calibration of the Nimbus-7, SMM and ERBS sensors were respectively reported as $\pm 0.5 \%, \pm 0.1 \%$, and $\pm 0.2 \%$. These values are about a factor of ten larger than the reported sensor stabilities.

The periodicity of various solar phenomena have frequently been analyzed using Fourier transforms. However, the observed periods generally vary with the particular stage of solar activity and to some extent with the type of solar phenomena being considered $^{12}$. Bouwer ${ }^{13}$ used a procedure termed dynamic power spectral analysis to examine the variation of the observed periods with time for several solar parameters. He analyzed the SMM irradiance but not the longer Nimbus-7 measurements. Earlier Fourier analyses of the Nimbus-7 measurements ${ }^{12,14}$ utilized the older, noisier data products. In the present study we use the new improved version of the dataset ${ }^{9}$ and concentrate on short term variations with periods ranging from a few days to 30 or 40 days.

The daily averaged Nimbus-7 solar irradiance measurements are shown in Fig. I(a) for the period November 1978 January 1993. There is a gap in the summer of 1992 when no measurements could be taken. The downward spikes in the irradiance occur when large sunspot groups face the Earth. Smaller upward spikes occur when bright faculae face the Earth. At low solar latitudes, the synodic rotation period is about 27.3 days; this period increases somewhat at higher latitudes. Short term periodicities are determined by the lifetimes and relative positions of the excited regions combined with the synodic rotation period. At sunspot maximum. excited regions are common and may persist for several solar rotations. At sunspot minimum, excited regions are rare and may last only a few days. In Fig. 1(a). the peaks of cycles 21 and 22 appear respectively on the left and right sides.

Wavelet transform of the Nimbus-7 solar irradiance measurements with Morlet wavelet is shown in Fig. 1(b). The dilation parameter a varies logarithmically from 4 (5.0 days, top of image) to 64 ( 80.4 days, bottom of image). Highest magnitudes are darkest. The general features in the solar irradiance measurements shown in Fig. 1(a). especially for the downward spikes, are seen to match well with the wavelet transform in Fig. 1(b). The present wavelet analysis of the Nimbus-7 data identifies in time and frequency, transient and periodic irradiance fluctuations ranging in period from 5.1 to 37.5 days.

No strong events appear in the relative quiet sun period from June 1984 through 1987. During sunspot maximum, it is common for two excited regions to appear about $180^{\circ}$ apart on the solar disc. This accounts for a large number of periods about 13.7 days long. The eventual fading of one of the excited regions or the addition of others will of course modify the calculated period. Periods of about 27.3 days arise from excited areas which give strong signals for two or more solar rotations. At times, two periods may occur at about the same time. Bouwer", using dynamic power spectral analysis. relates the total irradiance variation to a number of other solar parameters, such as sunspot information. the $C a-K$ plage index. $X$-ray measurements. etc. An advantage of wavelet analysis over the previous methods is a better time localization of these features. A more detailed analy sis of the dominant peaks in the wavelet transform for both vimbus- 7 and $5 . M 1$, is given in a previous study ${ }^{15}$. 
South Hemisphere which is mostly covered by oceans, there does not appear correlation between the solar eycle length and the temperature time series (Fig. 6).

\section{SUMMARY AND DISCUSSION}

The wavelet transform of the long-term solar irradiance dataset clearly shows the 11-year solar irradiance cycle (Fig. 2 b). However, in the long-term temperature datasets analyzed in this paper, there is no indication that there is any 11 -year cycle, either in phase or out of phase with the solar cycle. This does not necessarily imply that the Earth 's climate is not affected by the solar cycle; rather it simply reflects the complex nature of the Earth's response to climate forcings.

The variation in solar radiation of the Sun's 11 -year cycle is approximately $0.1 \%$. One may consider such a small variation insignificant. The long term variation of the Earth's orbital characteristics around the Sun. such as eccentricity and orientation, however, is also about $0.1 \%$. It has been hypothesized that a persistent variation of this magnitude could alter the temperature distribution on the Earth surface and could have led to the onset of Ice Ages in the past.

An indication of the sun-climate connection is manifested in the correlation between the solar cycle length obtained from the WT and the North Hemisphere temperature record (Fig. 7). For the South Hemisphere which is mostly covered by oceans, there does not appear such correlation.

A big uncertainty in detecting climate change comes from the Earth's oceans, which cover approximately $70 \%$ of the Earth's surface. The thermal inertia of the oceans is tremendous, as a depth of $2.5 \mathrm{~m}$ of water column has the same heat capacity of the entire atmospheric column. Except for the upper, mixed layer of the oceans, there are still too many unknowns about the dynamics of oceans in climate changes. There could be a very long time lag before any man-made or natural forcings manifest themselves as climate changes. Unfortunately, this also makes it very difficult to associate any climate changes with a climate forcing. The apparent absence of correlation between the solar cycle length and the South Hemisphere temperature anomalies may also be due to the influence of the oceans.

It has also been demonstrated ${ }^{20,21}$ before that the Earth is not insensitive to changes in solar radiation. The upper stratospheric ozone increases as the UV in the solar radiation increases, and it leads to stratosphere cooling. Since the stratosphere is coupled to the troposphere, variation of solar radiation inevitably affects the lower atmosphere and the Earth's surface. It has been discovered that the geopotential heights have shown some oscillation with a 10 to 12 years cycle. Since the geopotential height is a function of temperature. it may be the evidence that the solar radiation variation, while small, is directly affecting the climate.

The interaction among the elements in the Earth system - land, oceans, and atmosphere - under the external and internal forcings give out very complex signals. The periodicity of the forcings or signals could range from as short as diurnal to as long as a hundred thousand years. A period in the signal may also migrate as the various elements in the weather or climate system interact. Since wavelet transform can analyze multi-scale phenomena that are both localized in frequency and time, it is a very useful technique for detecting. understanding and monitoring climate changes.

For future studies, the time lag between the solar cycle length distribution extracted from wavelet transforms and the temperature anomalies time series will be analyzed. Hopefully this will shed some light on the mechanism of climate change responding to solar radiation variation. Datasets such as ozone and geopotential heights distributions will be analyzed to investigate the regional effects due to solar cycle. The possibility of using adaptive wavelet transform ${ }^{22}$ to improve the interpretation will also be explored.

\section{REFERENCES}

1. D. V. Hoyt and K. H. Schatten. "A discussion of plausible solar irradiance variations 1700-1992". I. Gerophrs. Res. Vol. 98. pp. 18895-18906. 1993. 

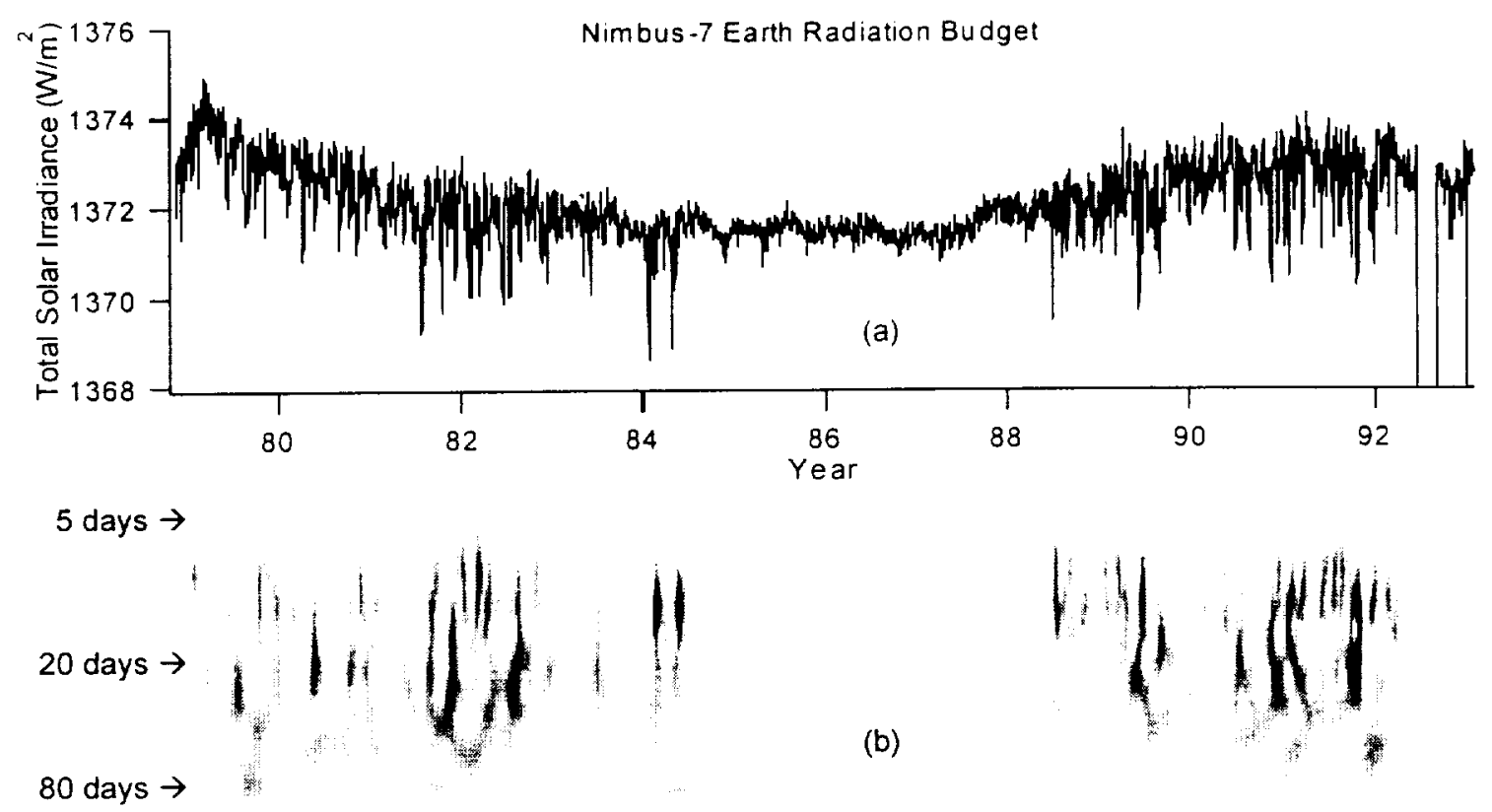

Figure 1. (a) 14 years of daily total solar irradiance measured by the Nimbus-7 Earth Radiation Budget instrument between November 1978 and February 1993. (b) The wavelet transform (period vs. time) of the time series in (a). Black is highest intensity, white is lowest.

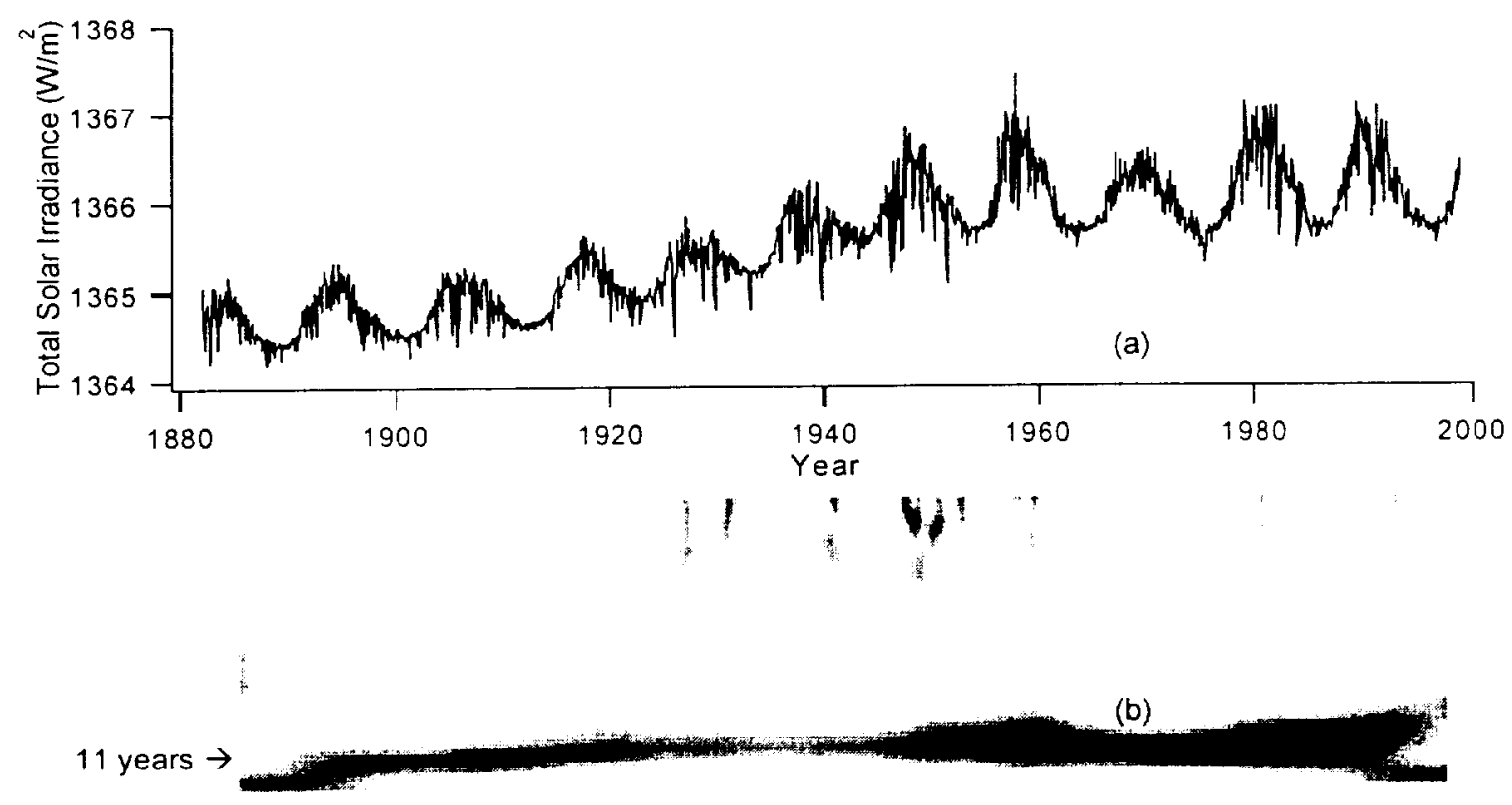

Figure 2. (a) 117 years of monthly solar irradiance record compiled by J. Lean. (b) The wavelet transform of the data. The dark band indicates the 10-12 ? ears solar cycle. 


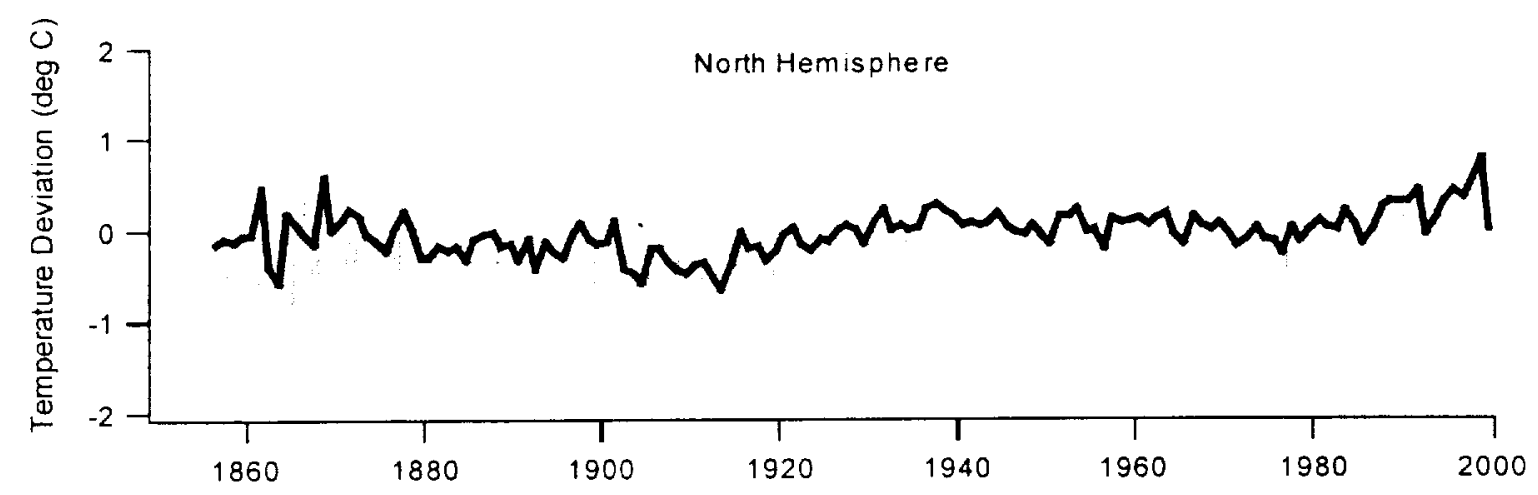

Figure 5. 144 years of monthly temperature deviation for the North Hemisphere compiled by the Climate Research Unit, Great Britain. The heavy curve represents the yearly July temperature. The annual cycle is clearly indicated in the computed wavelet transform (not shown here), but no 11 -year cycle is revealed.

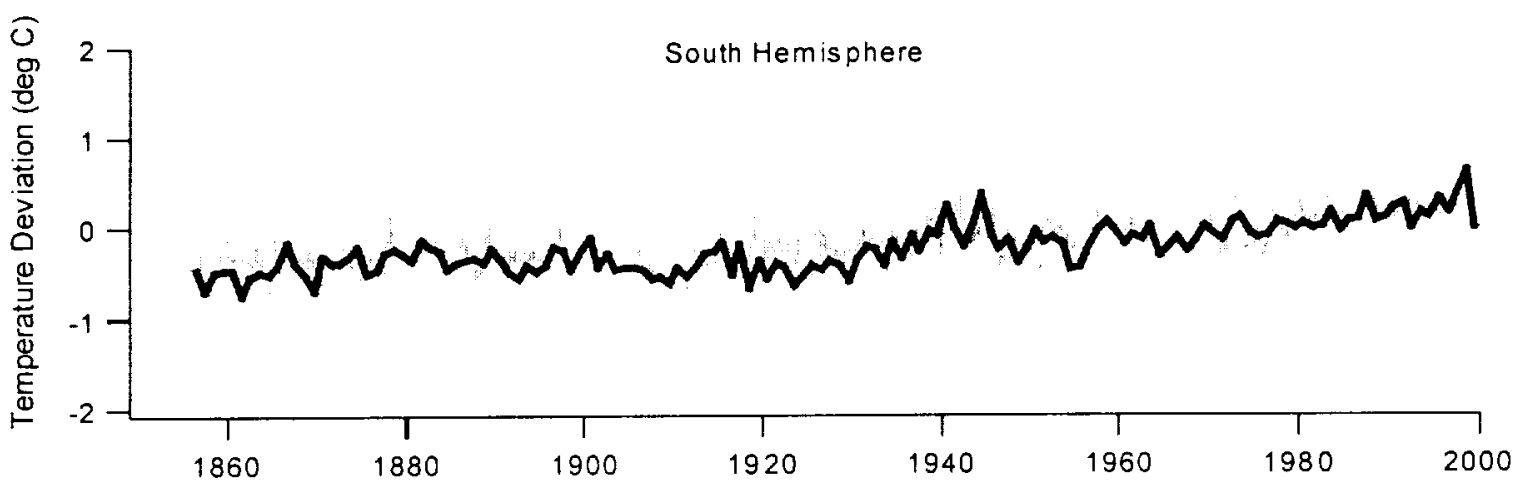

Figure 6. 144 years of monthly temperature deviation for the South Hemisphere compiled by the Climate Research Unit, Great Britain. The heavy curve represents the yearly July temperature. The annual cycle is clearly indicated in the computed wavelet transform (not shown here), but no 11 -year cycle is revealed. 